FACTA UNIVERSITATIS

Series: Mechanical Engineering Vol. 17, N² 2, 2019, pp. 149 - 159

https://doi.org/10.22190/FUME190511022G

Original scientific paper

\title{
CONTACT OF MULTI-LEVEL PERIODIC SYSTEM OF INDENTERS WITH COATED ELASTIC HALF-SPACE
}

\author{
Irina G. Goryacheva, Elena V. Torskaya
}

Ishlinsky Institute for Problems in Mechanics, Russian Academy of Sciences, Russia

\begin{abstract}
The contact of a periodic system of spherical indenters of different heights and radii of curvature with two-layered elastic half-space is considered. Numericalanalytical method is developed to determine contact pressure distribution and internal stresses taking into account mutual effect of contact spots. The results for relatively hard and soft coatings are analyzed for different values of input parameters: nominal pressure, contact density, coating thickness.
\end{abstract}

Key Words: Coatings, Discrete Contact, Contact Density, Internal Stresses

\section{INTRODUCTION}

The widespread use of coatings in different mechanisms actualizes the study of contact and internal stresses and contact fatigue damage accumulation inside the coated bodies. Surface roughness of counterbody is one of the parameters which influence contact characteristics (the pressure distribution and the real contact area) and stresses inside the coating and the base. For the case of cycling loading stress concentration near contact spots leads to the different types of the coating failure.

Roughness in contact problems is often modeled by periodic system of indenters to analyze mutual effect for different model geometry and to study real contact area and the depth of penetration as a function of average load applied to the period. 2-D periodic contact problems were studied mostly by analytical methods; the methods and results are reviewed in different books and papers, for example in [1]. The mutual effect for uncoated elastic solids was studied in [2] both for one-level and multi-level 3-D periodic systems of indenters penetrating into elastic half-space. The most recent studies of periodic contact problems for homogeneous and transversely isotropic elastic half-space [3-5] consider saturation

Received May 11, 2019 / Accepted July 03, 2019

Corresponding author: Irina G. Goryacheva

Affiliation: Ishlinsky Institute for Problems in Mechanics of the Russian Academy of Sciences, 119526

Moscow Prospect Vernadskogo,Russia

E-mail: goryache@ipmnet.ru 
effect in contact of periodic wavy surface [3] or adhesive contact for a system of indenters of different shape and transversely isotropic half-space [4].

The discrete contact problems for the coated elastic bodies were studied both for normal and sliding contacts in [6-9]. The coating thickness related to geometrical parameters of roughness is specific characteristic, which influences contact and internal stress distributions. Two approaches to model the discrete contact taking into account the mutual contact effect were developed almost at the same time. Periodic one-level system of spherical indenters penetrating into the two-layered elastic half-space was considered in [6]; 2-D contact problem for a measured profile of counterbody was solved in [7]. The same models of discrete contact were used to take into account roughness in 3D macro contact problem solution $[8,9]$; in both studies friction is also considered. For thick coatings some results from [2], which are mostly analytical, can be used for verification of semi analytical and numerical solutions of the similar counter body microgeometry.

In this study a multi-level periodic contact problem for a two-layered elastic halfspace is considered. Each level has particular geometry of indenters which are uniformly space distributed. The mutual effect of contact spots is taken into account. The model is used to calculate contact and internal stresses for relatively hard and soft coatings and to analyze the effect of microgeometry parameters (space distribution of asperities and their microshapes) on contact characteristics and internal stress distributions.

\section{PROBLEM ForMULATION AND THE METHOD OF SOLUTION}

\subsection{Problem formulation}

Two-layered elastic half-space is considered in contact with a periodic system of rigid indenters (asperities) uniformly distributed at $k$ height levels. In the cylindrical coordinate system related to each indenter, the shape of the indenter is described by the following function:

$$
z=f_{m}(r)+h_{m}, \quad m=1 \ldots k
$$

Here functions $f_{m}(r)$ and $h_{m}$ describe the shape and the height of the asperity of the m-th level respectively, $r$ is the radial coordinate of the polar system coordinates with the center at the point of initial contact of the asperity.

In contact interaction the areas of contact regions $\omega_{i m}$ are different for the contact spots of various levels (index $i$ indicates the fixed asperity of the $\mathrm{m}$-th level), and the asperities come into contact at definite penetrations of the whole system corresponding to their space location. The example of the contact spots distribution at the surface is presented in Fig. 1 for the case $k=3$. In this case the indenters are located at the nodes of the hexagonal lattice, and $l$ is the lattice period.

At the first step we introduce the polar system of coordinates at the layer surface with the center at the point of initial contact of the fixed highest asperity (see Fig.1). The centers of the other contact spots $\omega_{i m}$ have radius-vectors $\bar{r}_{i m}$. The axis $\mathrm{Oz}$ is perpendicular to the layer surface and directed inside two-layered half-space. Under the assumption that the shear stresses within the contact spots are negligibly small, the boundary conditions at the surface of the layer $(z=0)$ are the following: 


$$
\begin{aligned}
& w\left(\left|\bar{r}-\bar{r}_{i m}\right|\right)=f_{m}\left(\left|\bar{r}-\bar{r}_{i m}\right|\right)+h_{m}-\delta, \quad \bar{r} \in \omega_{i m}, \\
& \sigma_{z}^{(1)}=0, \quad \bar{r} \notin \omega_{i m}, \quad m=1 . . k, \quad i=1,2, \ldots \infty, \\
& \tau_{x z}^{(1)}=0, \quad \tau_{y z}^{(1)}=0, \quad 0 \leq|\bar{r}|<\infty
\end{aligned}
$$

where $\delta$ is the system penetration.

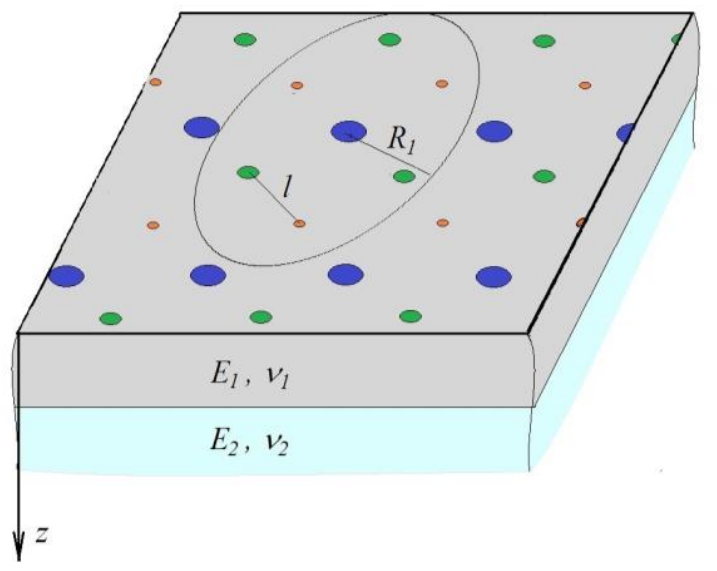

Fig. 1 Scheme of the contact for the system of indenters located at 3 height levels

Boundary conditions at the layer - half-space interface $(z=h)$ correspond to the case of perfect coating-substrate adhesion:

$$
\begin{array}{lll}
\sigma_{z}^{(1)}=\sigma_{z}^{(2)}, & \tau_{r z}^{(1)}=\tau_{r z}^{(2)}, & \tau_{\theta z}^{(1)}=\tau_{\theta z}^{(2)}, \\
w^{(1)}=w^{(2)}, & u_{r}^{(1)}=u_{r}^{(2)}, & u_{\theta}^{(1)}=u_{\theta}^{(2)}
\end{array}
$$

In Eqs. (2) and (3) $\sigma_{z}^{(j)}, \tau_{r z}^{(j)}, \tau_{\theta z}^{(j)}$ are normal and shear stresses, and $w^{(j)}, u_{r}^{(j)}, u_{\theta}^{(j)}$ are normal and tangential displacements ( $j=1$ for the coating, $j=2$ for the substrate). Contact zones $\omega_{i m}$ under the asperities are initially unknown. Note, that instead of the conditions of perfect adhesion at the interface, the conditions of complete or incomplete sliding can be considered using the approach developed in $[10,11]$.

Using the localization principle [2], we take into account the real pressure distribution only at the fixed number of asperities inside the circle of radius $R_{1}\left(|\bar{r}| \leq R_{1}\right)$, which includes a definite number of asperities of all $k$ levels. For known densities $\bar{Q}_{c}$ of the asperities distributions for each level $(c=1,2 \ldots, k)$ radius $R_{1}$ is calculated from the relation (for $k=1$ )

$$
R_{k}^{2}=\frac{1}{\pi}\left(\sum_{c=1}^{k} \frac{\eta_{c}}{\bar{Q}_{c}}+\frac{1}{\bar{Q}_{k}}\right)
$$

Here $\bar{Q}_{c}$ is the density of indenters of level $n, \eta_{c}$ is the number of indenters of level $c$ inside the circle. Action of indenters outside the circle is replaced by constant average pressure $\bar{p}$. Inside the circle unknown contact pressure distributions $p_{c}(r, \theta)$ act within the 
contact spots under each indenter (here $(r, \theta)$ are the polar coordinates related to the center of the $c$-th indenter). To satisfy the equilibrium condition we use the following relation:

$$
\bar{p}=\sum_{n=1}^{k} \bar{Q}_{n} \iint_{\omega_{n}} p_{n}(r, \theta) d r d \theta
$$

Note that due to the mutual effect the asymmetric contact pressure distribution under the axisymmetric indenter occurs, and we take into account this effect in contact problem formulation and solution.

The boundary element method together with the iterative procedure is used to solve the contact problem with unknown contact regions. Contact pressure is presented as a stepwise function being constant inside each element. We choose the size of a square element so that their number inside a contact region is large enough to provide convergence of the iterative procedure.

\subsection{Calculation of influence coefficients for the boundary element method}

To use the boundary element method, the displacement of the two-layered elastic halfspace surface loaded by a pressure $q$, uniformly distributed inside a square $2 a \times 2 a$, must be calculated from the following boundary conditions at the layer surface $(z=0)$ :

$$
\begin{aligned}
& \sigma_{z}=-q,|x| \leq a,|y| \leq a \\
& \sigma_{z}=0, \quad|x|>a,|y|>a \\
& \tau_{x z}=0, \tau_{y x}=0
\end{aligned}
$$

In the case of two-layered elastic half-space stresses and displacements of the layer surface can be calculated by using the method based on double Fourier transforms. In particular, normal displacements of the surface are determined by the following relation [11]:

$$
w^{\prime}\left(x^{\prime}, y^{\prime}, 0\right)=-\frac{q}{2 G_{1}} \int_{0}^{\pi / 2} \int_{0}^{\infty} \Delta(\gamma, \varphi, \lambda, \chi) \cos \left(x^{\prime} \gamma \cos \varphi\right) \cos \left(y^{\prime} \gamma \sin \varphi\right) d \gamma d \varphi
$$

Here $x^{\prime}, y^{\prime}$ and $w^{\prime}$ are dimensionless coordinates and normal displacement, respectively, $G_{1}$ is the shear modulus of the layer, $\chi=E_{1}\left(1+v_{2}\right) / E_{2}\left(1+v_{1}\right)$ is the relation of the elastic modules of the layer and the substrate, $\gamma, \varphi$ are the internal coordinates in the space of double Fourier transforms, $\lambda=h / a$ is dimensionless layer thickness. Function $\Delta(\gamma, \varphi, \lambda, \chi)$ represents normal displacements in the space of double Fourier transforms. It is obtained from the boundary conditions (3) and (6) using representations of stresses and displacements by a biharmonic function and double Fourier transforms of constant pressure $q$. It makes possible to reduce the problem to linear system of functional equations [11]. The solution of the system provides particularly the analytical representation of $\Delta(\gamma, \varphi, \lambda, \chi)$. As the dependence of normal displacements on the value of the constant pressure in Eq. (7) is linear, it can be used to find influence coefficients in the boundary element method. 


\subsection{Calculation of contact characteristics}

The boundary element method for a contact problem solution is described in many papers. In [6] it was developed to study the discrete contact problem with mutual effect of contact spots.

To solve the contact problem formulated in Eqs. (2) and (3), we used the following steps. At the first step, we place the center of the circle, in which we are looking for a real distribution of pressures, in the center of the highest indenter and calculate the surface shape $g(x, y)$ inside the circle with radius $R_{1}$, Eq. (4)), due to constant nominal pressure $\bar{p}$ outside the circle. The value of $\bar{p}$ at this step is small enough to provide only the contact of the indenters of the first level. Function $g(x, y)$ is used then in formulation of the contact conditions to calculate the pressure distribution under the indenters of the first level and the normal displacements outside the contact spots. Then we increase nominal (average) pressure controlling the displacements under indenters of the next level.

For pressures $\bar{p}_{2} \geq \bar{p} \geq \bar{p}_{1}$ the indenters of the second level come into contact. Then we take the origin of coordinates under the center of indenter of the second level. For calculation of the contact pressure under the indenters of the second level, we take into account the real contact pressure distributions at the contact spots of the first and second levels, and the nominal pressure outside the circle of radius $R_{2}$, Eq. (4), and use the iteration method. Using similar procedure for each $i$-th level we calculate the contact pressure distribution for each level of indenters, normal forces acted at indenters of each level and their redistribution if the nominal pressure increases.

Additional displacement $w_{a}$ of the periodical system of indenters is also calculated. This value indicates the displacement due to surface microgeometry, and it is calculated from the relationship [2]:

$$
w_{a}=\sum_{i=1}^{k} F_{\omega_{i m}}\left[p_{i}(r, \theta)\right]-F_{R_{i}}[\bar{p}]
$$

Here function $F_{\omega_{i m}}$ indicates the surface displacement under the highest indenter due to real contact pressures distribution under asperities located inside the circle of radius $R_{1}$, $F_{R_{i}}$ is related to the displacement caused by average pressure $\bar{p}$ distributed within the circle of radius $R_{1}$.

The dependence of the additional displacement of the periodical system of indenters on its geometrical characteristics is also analyzed.

\section{RESULTS AND DISCUSSION}

Calculations have been completed for the three levels periodic system of spherical indenters with the given heights. The indenters were located at the points of the hexagonal lattice with distance $l$. The indenter's shape at the $i$-th height level was spherical ( $r_{i}$ is the radius of curvature of the indenter near the point of initial contact). Note that the model under consideration is valid only for small deformations, so the saturation effect cannot be analyzed within the framework of this study. 
The dependence of the contact characteristics on the following model parameters has been analyzed:

- coating thickness $h$;

- Young modulus $E_{j}$ and Poisson ratio $v_{j}$ for coating $(j=1)$ and substrate $(j=2)$ materials;

- lattice period for the system of indenters, 1 ;

- radius of indenters (for each level), $r_{i}$;

- heights of indenters of each level $h_{i}$.

The following dimensionless parameters have been introduced:

- dimensionless radius of indenters for $i$-th level $(i=1,2,3)$,

$$
r_{i}{ }^{\prime}=r_{i} / l
$$

- dimensionless contact pressure distribution for i-th level

$$
p_{i}{ }^{\prime}(x, y)=p_{i}(x, y) / E_{2}
$$

- dimensionless average pressure

$$
\bar{p}^{\prime}=\bar{p} / E_{2}
$$

All internal stresses are also related to $E_{2}$. Dimensionless parameter $\chi$, which characterizes relative compliance of the coating, was introduced in the previous chapter. We do not use special letter symbols for the dimensionless thickness of the coating and the difference in height, but they are related to the lattice period.

The contact characteristics calculations for the system of indenters with three height levels contacting with the relatively hard coating bonded to the elastic base have been performed. In step-by-step solution with the increase of the average pressure first we have only one level of indenters in contact with the coating surface. For the chosen space distribution of indenters: $\left(h_{1}-h_{2}\right) / l=\left(h_{2}-h_{3}\right) / l=0.01$ mutual effect for the first level was negligible, the pressure under each indenter was axisymmetric. For relatively large average pressure three levels are in contact, the mutual effect becomes stronger. Fig.2 illustrates the contact pressure distribution (Fig. 2a) and the contact spot configuration (curve 1 in Fig. 2b) for this case. We have got not circular contact zone, especially for the indenters of the lowest level. Mutual effect can be evaluated by comparison of curves 1 and 2 in Fig. 2b; the last one was calculated for the same input parameters, but neglecting the surface deformation due to the penetration of nearby indenters of the first and second levels. Maximum value of the contact pressure in Fig. $2 \mathrm{a}$ is $\left(p_{3}{ }^{\prime}\right)_{\max }=0.07$.

Fig. 3 illustrates the contact pressure distributions calculated for relatively soft coatings characterized by the different Poisson ratio. The chosen cross section provides the maximum size of the contact spot. The difference of maximal and minimal distance from the center of the contact zone to the boundary, which characterizes asymmetry due to the mutual effect, is 12 percent for the third level (curve 3); the difference is smaller for the first and the second levels. Contact spots for the first level hereinafter are almost axisymmetric. Comparison with pressure distributions obtained for isolated indenters loaded by the same forces (dashed lines in Fig. 3a,b) leads to conclusion that for the case of relatively soft low-compressive coatings $\left(v_{1}=0.45\right)$ mutual effect is strong (see also Table 1), but for $v_{1}=0.2$ it is negligible. 
a)

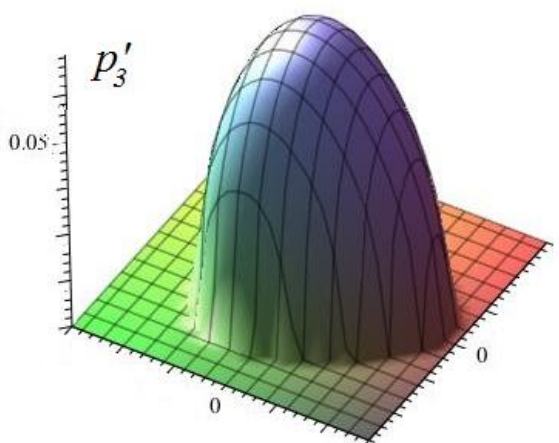

ig. 2 Contact pressure distribution (a) and contact spot (b) for the $3^{\text {rd }}$ level of indenters: $\left.\chi=2, h / l=0.5,\left(h_{1}-h_{2}\right) / l=\left(h_{2}-h_{3}\right) / l=0.01, \bar{p}^{\prime}=0.06\right), v_{1}=0.2, v_{2}=0.3, r_{1}{ }^{\prime}=r_{2}{ }^{\prime}=r_{3}{ }^{\prime}=0.8$

a )

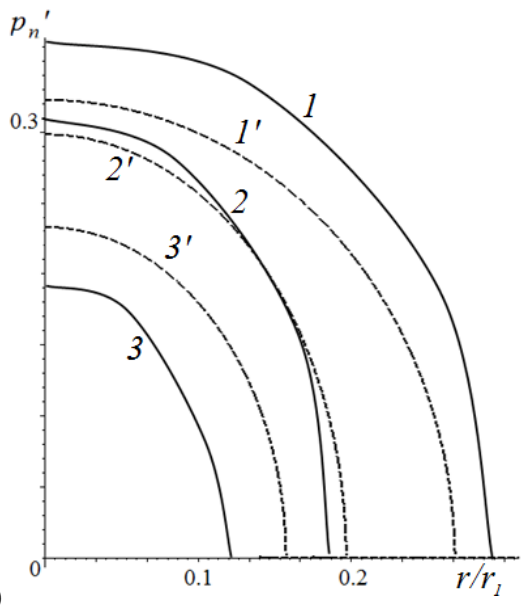

b

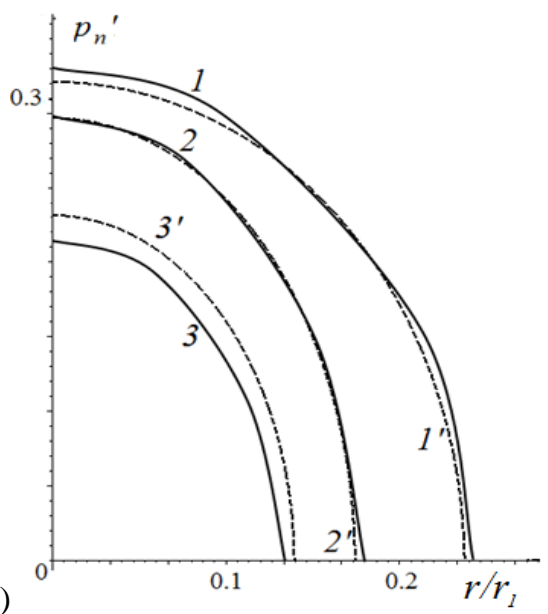

Fig. 3 Pressure distribution under indenters of levels 1-3 (curves 1-3 respectively) for relatively soft coatings $\chi=0.5, h / l=0.5$ with $v_{1}=0.45$ (a), $v_{1}=0.2$ (b): $v_{2}=0.3$, $\left(h_{1}-h_{2}\right) / l=\left(h_{2}-h_{3}\right) / l=0.01, r_{1}{ }^{\prime}=r_{2}{ }^{\prime}=r_{3}{ }^{\prime}=0.8, \bar{p}^{\prime}=0.008$

Table 1 Maximal values of contact pressure related to $E_{2}$ (results from Fig. 3a)

\begin{tabular}{ccccc}
\hline & \multicolumn{2}{c}{$v_{1}=0.45$} & \multicolumn{2}{c}{$v_{1}=0.2$} \\
\cline { 2 - 5 } & $\begin{array}{c}\text { Calculation with } \\
\text { mutual effect }\end{array}$ & Isolated indenters & $\begin{array}{c}\text { Calculation with } \\
\text { mutual effect }\end{array}$ & $\begin{array}{c}\text { Isolated } \\
\text { indenters }\end{array}$ \\
\hline Level 1 & 0.356 & 0.312 & 0.320 & 0.311 \\
Level 2 & 0.298 & 0.289 & 0.288 & 0.287 \\
Level 3 & 0.191 & 0.232 & 0.213 & 0.230 \\
\hline
\end{tabular}


Fig. 4a illustrates the results of calculation of the additional displacement function (8) for relatively hard coating of various thicknesses: $h / l=0.12$ (curves $1,1^{\prime}$ ) and $h / l=0.24$ (curves 2,2') in the cases of three level model (curves 1 and 2) and one-level model (curves 1' and 2').

The results indicate that in the case of hard coatings at the softer substrate the additional displacement for three level asperities distribution is higher than for the one level model under the same nominal pressure. Decreasing the coating thickness leads to increasing of the additional displacements.

It is interesting to note that for the case of relatively thick coatings the difference between curves 2 and 2' (representing different models) is greater than for thinner coatings (curves 1 and 1 ' respectively).

To analyze the mutual effect we calculated the additional displacement function ignoring the surface displacements outside the contact regions (neglecting mutual effect), but taking into account equilibrium conditions. The results of calculations are presented in Fig. 4b (solid curves 1 and 2 are the same as in Fig. 4a, dashed curves are calculated neglecting mutual effect). There is an essential difference between the curves. The penetration calculated without mutual effect is greater. So neglecting the mutual effect gives the overestimate of additional displacement due to roughness.

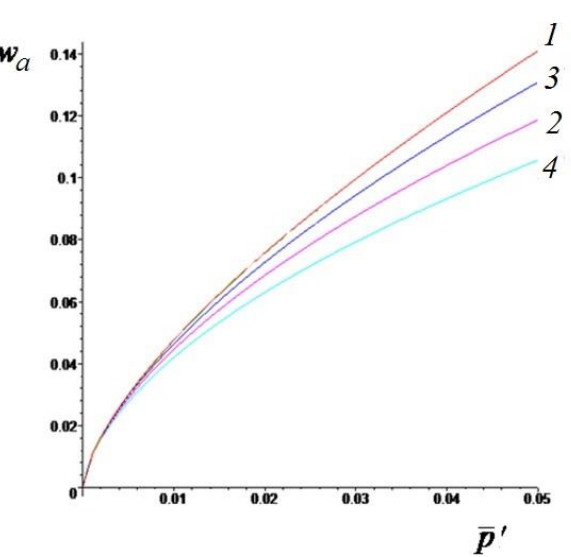

b)

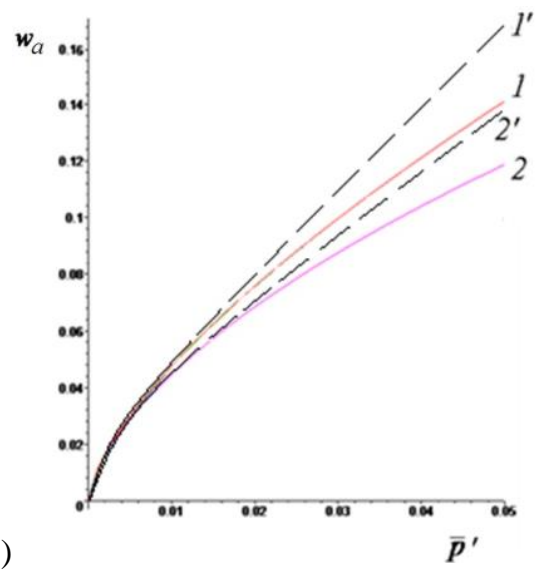

Fig. 4 Dependence of the additional normal displacements on nominal pressures for one-level and three-level models (a) and comparison of the dependences for three level model with ones (curves 1' and 2') calculated neglecting mutual effect (b): $h / l=0.12$ (curves 1,3$), h / l=0.24$ (curves 2,4$),\left(h_{1}-h_{2}\right) / l=\left(h_{2}-h_{3}\right) / l=0.01$ (curves 1,2 ), $\left(h_{1}-h_{2}\right) / l=\left(h_{2}-h_{3}\right) / l=0$ (curves 3,4), $\chi=02, v_{1}=0.22, v_{2}=0.3, r_{1}{ }^{\prime}=r_{2}{ }^{\prime}=r_{3}{ }^{\prime}=0.8$

The results presented in Fig. 5 give a possibility to analyze the influence of nominal pressure on contact characteristics (maximal contact pressure and contact size) for two types of microgeometry models: one-level model with period $\sqrt{3} l$ (curves 1), three-level model with the same period $\sqrt{3} l$ for each level (curves 2). The results indicate that the radius of contact spot and maximal contact pressure increases with increasing the nominal pressure. No difference exists between the curves 1 and 2 for small values of average pressure, when the second and third levels are not in contact. 
Curve 3 in Fig. $5 b$ illustrates the principal shear stress maximal value. The maximum is localized at the layer-substrate interface for the chosen model parameters.

Figs. 6 and 7 illustrate the influence of the contact density on the distribution of tensile-compressive and principal shear stresses under an indenter of the first level for the value of the nominal pressure $\bar{p}^{\prime}$, which provides three-level contact. All geometrical parameters are related here to the radius of first-level indenter, which allows us to use different value of $l$ associated with the contact density.
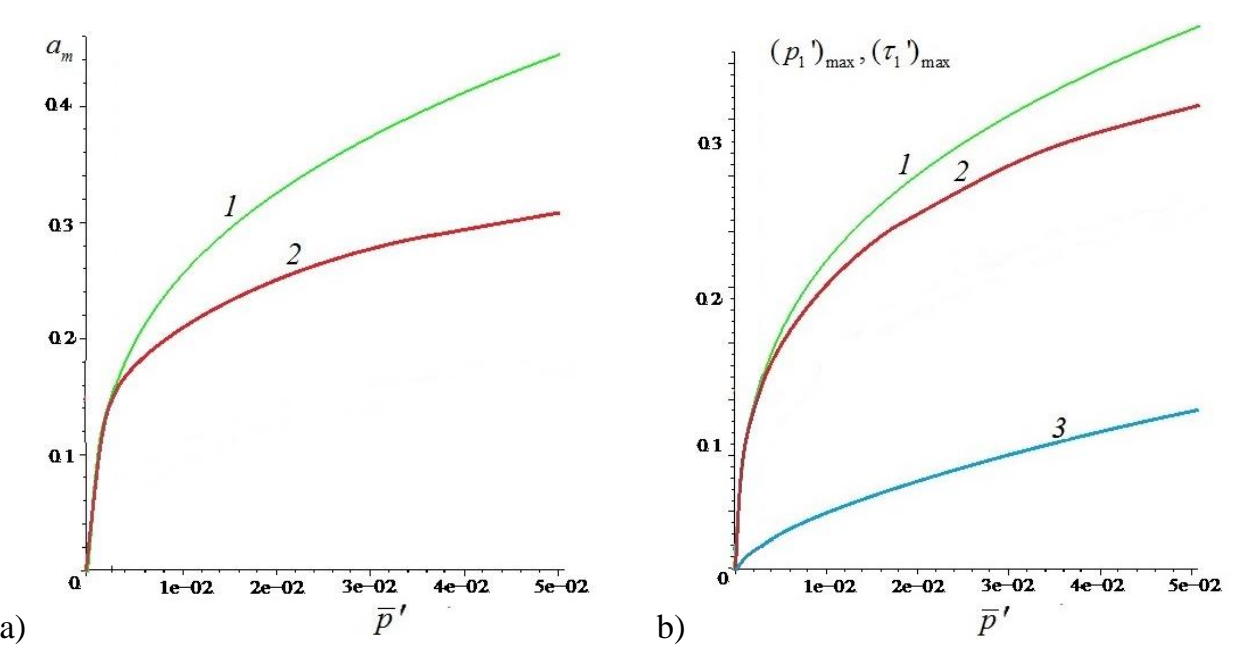

Fig. 5 Dimensionless maximal contact radius $a_{m}$ (a), maximal contact pressure $\left(p_{1}{ }^{\prime}\right)_{\max }$ (b, curves 1-2) and maximal values of the principal shear stress in the coating $\left(\tau_{1}{ }^{\prime}\right)_{\max }(\mathrm{b}$, curve 3 ) under first-level indenter as functions of nominal pressure; $\left(h_{1}-h_{2}\right) / l=\left(h_{2}-h_{3}\right) / l=0.06, r_{1}{ }^{\prime}=1.67, r_{2}{ }^{\prime}=1.25, r_{3}{ }^{\prime}=0.83$ (curves 1,3 ), one-level model with period $\sqrt{3} l$ and $r_{1}{ }^{\prime}=1.67$ (curves 2$) ; h / l=0.087, \chi=3, v_{1}=0.22, v_{2}=0.4$

We chose these stresses for analysis, because due to their concentration the coating delamination due to brittle fracture (large tension) or contact fatigue (high amplitude values of the principal shear stress) occurs. Since the space distribution of indenters influences essentially on the contact spot radius and the maximal contact pressure (see Fig. 5), the internal stress distribution depends also on the density and height distribution of asperities. The stress distributions presented in Figs. 6 and 7 are typical for relatively hard coatings: tension occurs at the surface near the boundary of contact, and at the coating-substrate interface for thicker coating; principal shear stresses concentrate at the surface-substrate interface, which is often initially damaged.

Fig. 7 illustrates the results of stress calculation for the same input parameters as used in Fig. 6. The values of tensile-compressive stresses (curves 1-3) are very different for the cases of high (b) and low (a) contact densities as at the surface and as at the interface. For the case of high contact density positive tensile stresses occur at the surface under the boundary of the contact zone (curve 1), in coating material at the interface under the center of the contact (curve 2), and only compression realizes in substrate material (curve 3). For the case of low 
density both for the surface and for the interface the curves have the same features, which are compression under the center and tension under the boundary of the contact zone. Principal shear stresses for both cases have interface maxima.

a)
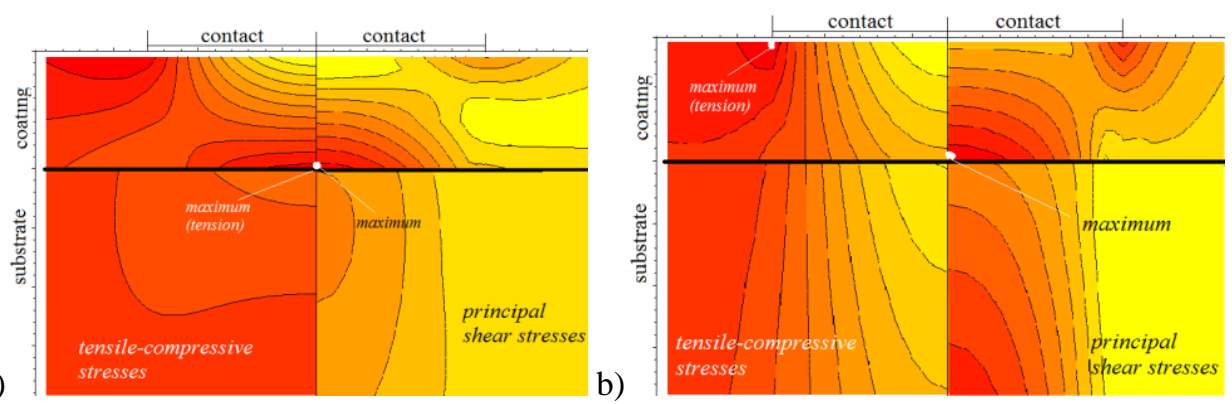

Fig. 6 Tensile-compressive and principal shear stresses under first-level indenter: $\chi=3$, $\bar{p}^{\prime}=0.002, v_{1}=0.22, h / r_{1}=0.023 v_{2}=0.4 ; r_{2} / r_{1}=0.75, r_{3} / r_{1}=0.5,\left(h_{1}-h_{2}\right) / r_{1}=\left(h_{2}-h_{3}\right) /$ $r_{1}=0.036, l / r_{1}=0.067$ (a) $l / r_{1}=0.6$ (b)

a)

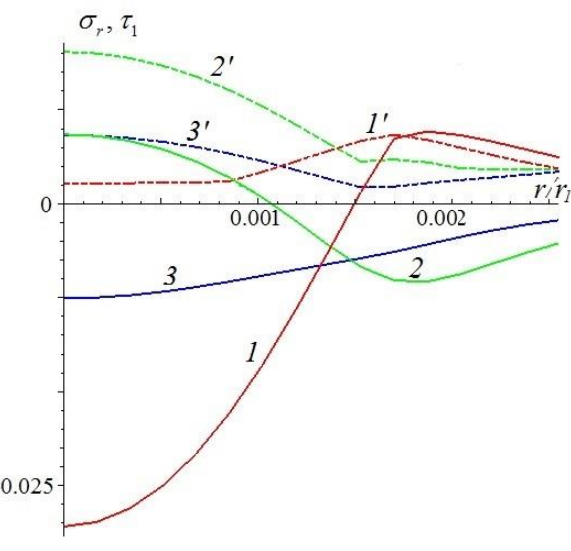

b)

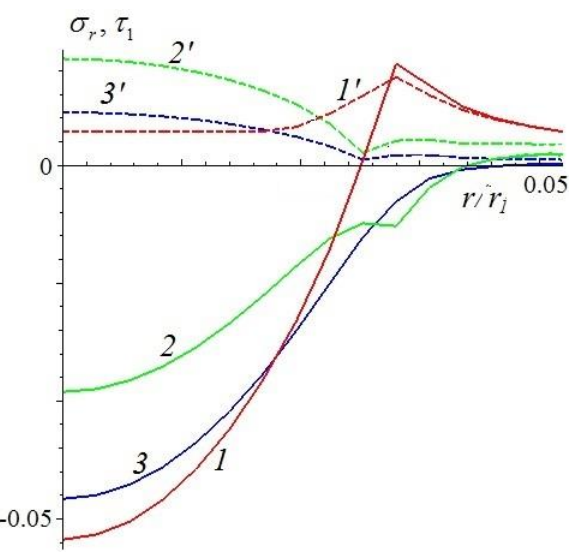

Fig. 7 Tensile-compressive $\sigma_{r}$ (curves 1, 2, 3) and principal shear $\tau_{1}$ (curves 1', 2', 3') stresses under first-level indenter: $\chi=3, \bar{p}^{\prime}=0.002, v_{1}=0.22, h / r_{1}=0.023, v_{2}=0.4$; , $r_{2} / r_{1}=0.75, r_{3} / r_{1}=0.5,\left(h_{1}-h_{2}\right) / r_{1}=\left(h_{2}-h_{3}\right) / r_{1}=0.036, l / r_{1}=0.067$ (a) $l / r_{1}=0.6$ (b) at the surface (curves 1, 1'), at the coating-substrate interface in coating (curves 2, 2') and substrate (curves 3, 3')

\section{CONCLUSIONS}

The numerical-analytical method of contact problem solution for multi-level periodic system of indenters and two-layered elastic half-space is developed. Contact pressure distributions under the indenters of different levels, internal stresses, and additional displacements as a function of average pressure are determined for different values of 
input parameters: nominal pressure, space distribution of indenters, their contact density, coating thickness and its relative to the base mechanical properties.

The mutual effect is analyzed for the case of relatively hard and soft coatings. The results indicate that for the case of thin soft coatings this effect is stronger for materials with relatively high value of Poisson ratio. For relatively hard thin coatings due to the coating bending the real contact pressure distribution and the real contact area strongly depend on the contact density and height distribution of indenters, which can be considered as the model of the asperities of the rough surface with regular roughness.

It follows from the results that the internal stresses in relatively hard coatings also depend essentially on the contact density and space distribution of asperities. Tensile-compressive and principal shear stresses are very different for the cases of low and high density both for the surface and for the interface. For the case of high contact spot density the tension of coating occurs at the layer-substrate interface, but for low density there is the compression. For small contact density, interface tension is realized not under but between indenters.

Based on the results it may be concluded that the mutual effect is stronger for hard coatings, than for the soft ones. The similar conclusion was made from the problem solution for the one-level asperity model in contact with the two-layered elastic half-space [6].

Acknowledgements: This work was carried out under the financial support of the Russian Foundation for Basic Research (grants N. 17-58-52030 (Russian-Taiwan) and 17-01-00352).

\section{REFERENCES}

1. Goryacheva, I.G., Martynyak, R.M., 2014, Contact problems for textured surfaces involving frictional effects, Proceedings of the Institution of Mechanical Engineers, Part J: Journal of Engineering Tribology, 228(7), pp. 707-716

2. Goryacheva, I.G., 1998, Contact Mechanics in Tribology, Dordercht: Kluwer Academic Publ, 344 p.

3. Yastrebov, V.A., Anciaux, G., Molinari, J-F., 2014, The contact of elastic regular wavy surfaces revisited, Tribology Letters, 56, pp. 171-183.

4. Argatov, I.I., Li, Q., Popov, V.L., 2019, Cluster of the Kendall-type adhesive microcontacts as a simple model for load sharing in bioinspired fibrillar adhesives, Archive of Applied Mechanics, 3, pp. 1-26.

5. Argatov, I.I., 2012, The contact problem for a periodic cluster of microcontacts, Journal of Applied Mathematics and Mechanics, 76, pp. 604-610.

6. Goryacheva, I.G.. Torskaya, E.V., 2003, Stress and fracture analysis in periodic contact problem for coated bodies, Fatigue and Fracture of Engineering Materials and Structures, 26(4), pp. 343-348.

7. Cole, S.J., Sayles, R.S., 1991, A numerical model for the contact of layered elastic bodies with real rough surfaces, Journal of Tribology, 11, pp. 334-340.

8. Torskaya, E.V., 2012, Modeling of frictional interaction of a rough indenter and a two-layered elastic half-space, Physical Mesomechanics, 15(3-4), pp. 245-250.

9. Nyqvist, J.T., Kadiric, A., Sayles, R.S., Ioannides, E., 2013, Three-dimensional analysis of multilayered rough surface contact, Proc. 5th World Tribology Congress, Torino, Italy.

10. Goryacheva, I.G., Torskaya, E.V., 2016, Modeling the influence of the coating deposition technology on the contact interaction characteristics, Mechanics of Solids, 51(5), pp. 550-556.

11. Nikishin, V.S., Shapiro, G.S., 1970, Space problems of the elasticity theory for multilayered media, Moscow: VTs AN SSSR, 260p. (in Russian). 